\title{
Selección de categorías para el estudio de la evolución de la competencia digital docente del profesorado en Educación Superior
}

\section{Selection of categories for the study of the evolution of teachers' digital competence in Higher Education}

\author{
Angelina Lorelí Padilla-Hernández \\ Universidad de Granada \\ angelina.loreli@gmail.com \\ Vanesa M.a Gámiz-Sánchez \\ Universidad de Granada \\ vanesa@ugr.es \\ M.aA Asunción Romero-López \\ Universidad de Granada \\ romerol@ugr.es
}

Recibido: $14 / 04 / 2018$

Aceptado: $18 / 06 / 2018$

Publicado: 29/06/2018

\section{RESUMEN}

La competencia digital docente (CDD) puede comprenderse como una competencia en evolución a lo largo de la trayectoria profesional del profesorado. Este es el tema de investigación de una tesis doctoral en curso, cuyo objetivo general es caracterizar la adquisición y el desarrollo en la práctica de la competencia digital de docentes universitarios para reconocer su influencia en su evolución profesional. En este artículo se presentan las categorías identificadas para el diseño de un guion de entrevista en profundidad acerca de la evolución de dicha competencia. Se realizó la revisión de literatura especializada que incluyó el análisis descriptivo de dos modelos sobre la CDD del profesorado universitario y de los instrumentos de seis estudios recientes del ámbito iberoamericano. La síntesis se organizó en tres apartados: las líneas específicas de la CDD en la docencia universitaria, áreas de la competencia digital relacionadas con el desempeño de los docentes, y aspectos para comprender la evolución de la CDD. Como resultado se desarrolló el listado de dimensiones, categorías e indicadores sobre la evolución de la CDD en Educación Superior. Por último, se discute el alcance y las limitaciones de este listado, que se someterá a un estudio de obtención de evidencias de validez mediante el juicio de expertos.

\section{PALABRAS CLAVE}

Competencia digital; Educación Superior; Competencias del profesorado; Desarrollo profesional docente; Narrativas personales.

\section{ABSTRACT}

Digital competence can be understood as an evolving competence throughout the professional career of teachers. This is the research topic of an ongoing doctoral thesis, whose general objective is to characterize the acquisition and development in practice of the teachers' digital competence of professors, to find out the influence on their professional evolution. This article presents the identified categories for the design of an in-depth interview guide about the evolution of digital competence in Higher Education teachers. After reviewing specialized publications, a descriptive analysis has been made about the digital competence of university teachers and the instruments used by six recent studies from the iberoamerican context. The synthesis was organized in three topics: specific areas of the teachers' digital competence in Higher Education, areas of the general digital competence related to the teaching process, and the aspects for understanding the evolution of this competence. As a result, a set of dimensions, categories and indicators focused on the evolution of the digital competence of Higher Education teachers was developed. Finally, the scope and limitations of this set are discussed, making it obvious that we need to evaluate it through an expert judge panel aimed to obtain content validity evidence. 


\section{KEYWORDS}

Digital competence; Higher Education; Teacher competencies; Teacher professional development; Personal narratives.

\section{CITA RECOMENDADA}

Padilla-Hernández, A. L., Gámiz-Sánchez, V. M. y Romero-López, M. A. (2018). Selección de categorías para el estudio de la evolución de la competencia digital docente del profesorado en Educación Superior. RIITE. Revista Interuniversitaria de Investigación en Tecnología Educativa, 4, 55-67. Doi: http://dx.doi.org/10.6018/riite/2018/327881

\section{Principales aportaciones del artículo y futuras líneas de investigación:}

- Enfatizar la necesidad de comprender el carácter evolutivo de la competencia digital docente (CDD) del profesorado universitario.

- Compartir la selección de categorías para la elaboración de una guía de entrevista en profundidad.

- Los hallazgos del estudio general podrán aportar al debate sobre los desafíos de la relación entre la Educación Superior y la cultura digital, desde una perspectiva crítica que valora las experiencias, el conocimiento y las reflexiones del profesorado.

\section{INTRODUCCIÓN}

En los últimos veinte años ha aumentado el interés por el rol del docente en el aprovechamiento de las tecnologías digitales por parte de educadores y profesores, investigadores y organismos nacionales e internacionales de política educativa. La competencia digital docente (CDD) constituye una vía de aproximación a este tema, pues aborda la competencia profesional de los docentes al incorporar tecnologías digitales en su labor. Además, es una línea de investigación actual en el ámbito de la Tecnología Educativa (Gisbert, González y Esteve, 2016).

Con respecto a la competencia digital docente en la Educación Superior, estudios realizados en los contextos mexicano y español reportaron varias necesidades formativas de los docentes en las dimensiones pedagógica, comunicativa y de colaboración en red, de investigación y desarrollo profesional (Carrera y Coiduras, 2012; Pozos, 2015; Prendes y Gutiérrez, 2013; San Nicolás, Vargas y Area, 2012). Nos encontramos ante un tema complejo pues si bien existe un potencial de transformación y de calidad educativa (Zabalza, 2003; Romero-López, 2017), el mero uso de tecnologías no supone la mejora educativa (Cobo, 2016; Coll, 2007). Además, la adecuada integración de tecnologías por parte de los docentes necesita condiciones profesionales, institucionales y políticas que la favorezcan (Pozos, 2015). Así, se requiere una mirada crítica de las prácticas y los discursos sobre la educación y las tecnologías (Selwyn, 2014).

Esta investigación en curso reconoce la necesidad de profundizar en la comprensión del desarrollo de la CDD del profesorado universitario. Reconocemos que existen diferentes perfiles docentes, así como experiencias en el uso de tecnologías en la Educación Superior. Observamos también que los programas de formación docente pueden no ser suficientes para responder a las demandas en relación a la CDD. Por medio de los relatos de vida queremos reconocer las reflexiones, beneficios y dificultades que docentes universitarios han experimentado a lo largo de su trayectoria a fin de dar sentido a las prácticas y recursos digitales. 
El propósito de este artículo es compartir la selección de categorías para la elaboración de un guion de entrevista en profundidad sobre la evolución de la CDD del profesorado universitario. Se consideraron dos objetivos: la delimitación de la CDD en Educación Superior y la organización de categorías para fundamentar el guion de entrevista de carácter abierto y a la vez con temas en común para abordar los relatos de los docentes participantes (Bolívar, Domingo y Fernández, 2001; Rodríguez, Flores y García, 1996). Enseguida presentamos un panorama del estado de la cuestión de interés para esta investigación.

\section{ESTADO DEL CONOCIMIENTO}

\subsection{La conceptualización de la competencia digital docente}

La competencia digital es un concepto reciente que refiere a las habilidades para el uso de tecnología que es clave en el debate acerca de los conocimientos que las personas necesitan en la era digital (Gallardo-Echenique, 2013). Definirla es una tarea compleja pues existen múltiples formas de entenderla y nombrarla (Ala-Mutka, 2011; Amorós, 2013; GallardoEchenique, Minelli de Oliveira, Marqués-Molias y Esteve-Mon, 2015; Gisbert et al., 2016). Esto se debe a que depende del contexto (Calvani, Carteri, Fini y Ranieri, 2008), la finalidad perseguida (Ala-Mutka, 2011), y de su enfoque (Amorós, 2013). Además, su naturaleza es cambiante por su vínculo con la emergencia del ámbito digital (Janssen et al., 2013).

En resumen, los conceptos coinciden en referir un conjunto de conocimientos, habilidades y actitudes que favorecen el uso crítico, responsable y creativo de las tecnologías digitales, para distintos propósitos, entre ellos el aprendizaje y el desarrollo profesional. En línea con la indagación de Janssen et al. (2013), entendemos la competencia digital como un concepto multifacético y plural que revela una red de relaciones entre distintos aspectos de la competencia, con un carácter procesual, actitudinal y reflexivo.

Un referente importante para los modelos de CDD en la Educación Superior son algunos marcos del ámbito internacional, como los estándares propuestos por el International Society for Technology in Education, (ISTE, 2008), los estándares en competencia TIC (Tecnologías de la Información y la Comunicación) para docentes de la Organización de las Naciones Unidas para la Educación, la Ciencia y la Cultura (UNESCO, 2011), ambos en proceso de actualización, y la línea de trabajo de la Comisión Europea (Vuorikari, Punie, Carretero Gomez, Van den Brande, 2016; Redecker, 2017). Así también existen propuestas de distintas universidades, como las recogidas por el New Media Consortium (Alexander, Adams Becker, Cummins y Hall Giesinger, 2017). Esta diversidad puede entenderse como una oportunidad de debate acerca de las limitaciones y alcances de cada modelo (Castañeda, Esteve y Adell, 2018), y también, una posibilidad de diálogo con el conocimiento que emerge de las experiencias de docentes universitarios.

En particular, resaltamos la propuesta del marco común de competencia digital docente elaborado por el Instituto Nacional de Tecnologías Educativas y Formación del Profesorado (INTEF, 2013, 2017), en base al marco DIGCOMP de la Unión Europea. Las cinco áreas de competencia que incluye son: información y alfabetización informacional, comunicación y colaboración, creación de contenido digital, seguridad y resolución de problemas.

\subsection{Competencia digital docente en Educación Superior}

Hemos constatado en la revisión de literatura que existen menos investigaciones dedicadas a la CDD del profesorado universitario en contraste con los estudios realizados sobre docentes de educación básica; esto indica la necesidad de investigar la forma en que son aprovechadas las tecnologías digitales o los estándares para su integración (Veletsianos, Kimmons y French, 2013; Mengual y Roig, 2012). 
Para identificar los aspectos que constituyen la CDD del profesorado universitario fueron revisados dos modelos específicos de la docencia universitaria. Ambos modelos se basan en una amplia revisión de marcos sobre competencia digital y CDD.

El primero de ellos fue el propuesto por Durán, Gutiérrez y Prendes (2016a) sobre la competencia TIC de docentes universitarios. El modelo está organizado en tres niveles consecutivos de adquisición de la competencia: bases de conocimiento como fundamento del uso de TIC, competencias relacionadas al diseño, implementación y evaluación, y aquellas para el análisis reflexivo y crítico de la acción con TIC (Prendes, 2010; Prendes y Gutiérrez, 2013). La competencia TIC se entiende como una "alfabetización múltiple compleja", que integra la competencia digital general de la ciudadanía y el "criterio pedagógico-didáctico" (Durán et al., 2016a, p. 99). Este trabajo forma parte de un proyecto más amplio de investigación que busca certificar dicha competencia mediante un instrumento de evaluación que incluye cuestionarios, recopilación de evidencias y una prueba práctica (Durán et al., 2016b).

Por su parte, Pozos (2015) realizó un modelo integrador con siete unidades competenciales que a su vez se desglosan en competencias, enmarcadas en el desarrollo profesional del profesorado universitario. En el marco de la Sociedad del Conocimiento, se entiende esta competencia como básica para el desarrollo del docente, que implica la gestión de la información, el trabajo en equipo y en red, y la responsabilidad social al integrar las tecnologías digitales en su labor. Se reconocen varias fases en el proceso del docente: acceso, adopción, adaptación, apropiación e innovación. Así también, el dominio de competencias se caracteriza por niveles, desde el latente o no desarrollado, hasta el nivel experto. El instrumento principal fue un cuestionario de autoevaluación que se aplicó a los docentes participantes para identificar el nivel competencial y las necesidades formativas (Pozos, 2015).

\subsection{Aspectos de interés para comprender la evolución de la CDD}

Una de las líneas de interés para comprender la evolución de dicha competencia es el desarrollo profesional docente (DPD). Desde una perspectiva holística, el DPD comprende los cambios que los profesores experimentan en su conocimiento y práctica a lo largo de su itinerario profesional, que es contextual, dinámico y complejo (Fernández, 1995; 2006). Estos cambios no son lineales, es decir, podrían significar mejoras, estancamientos o bien ser reversibles (Fernández, 1995). Tres aspectos integran el DPD desde la perspectiva biográfica narrativa: la trayectoria profesional, el perfil docente, y el ciclo vital (Fernández, 2006). Así también, el desarrollo profesional se inscribe en un contexto institucional y social (Santos Guerra, 2010).

En este sentido, la narrativa es una vía de acceso a las complejas interacciones entre los aspectos que influyen el desempeño docente (Kelchtermans, 1999). Como Fernández (2014) señala, los relatos de vida son una reconstrucción subjetiva que se estructuran desde una perspectiva temporal, integran influencias de ámbitos personales, públicos y de distintas facetas de la personalidad del docente y pueden hablar de diferentes planos contextuales (grupos, instituciones, espacios de interacción). Las historias de vida de los docentes es una línea de investigación con una amplia tradición (Abrahão y Bolívar, 2014; Bolívar, Domingo y Fernández, 2001; Connelly y Clandinin, 1995; Flores y Porta, 2012; Goodson, 2004).

\section{MÉTODO}

Con el propósito de fundamentar la elaboración del guion de entrevista en profundidad fue necesario identificar categorías para aproximarnos a la evolución de la CDD. Dos objetivos específicos se tuvieron en cuenta: delimitar la CDD del profesorado universitario y organizar las categorías relevantes para el guion de entrevista. Para ello, la revisión de literatura 
especializada aportó información sobre los conceptos clave de la investigación, los marcos generales y los modelos de la CDD en Educación Superior, así como los indicadores de los instrumentos utilizados por estudios sobre la CDD.

La revisión de literatura se realizó a partir de la consulta a las bases de datos Web of Science, ERIC, Scopus, IRESIE y EBSCO. Utilizamos varios descriptores y palabras clave, como: competencia digital o competencia TIC, alfabetización digital, profesor o docente, universidad o Educación Superior como campo de estudio, y su adecuación al idioma inglés. Éstos se eligieron por su precisión con el tema de investigación y de acuerdo a la revisión de los tesauros de IRESIE, ERIC y EBSCO pues en ocasiones los descriptores no coincidían con las palabras clave de la investigación. Por otra parte, el uso de estos descriptores se fue adaptando según el resultado de las búsquedas.

Se realizaron dos tipos de registro de este proceso. El primero tuvo un carácter general pues se documentó el número de resultados obtenidos por cada base y según la combinación de descriptores. El segundo se enfocó en la organización de los documentos recuperados al registrar la información general de cada artículo y sus características (año de publicación, tipo de trabajo, país y tema, entre otros) según la lectura del título y del resumen. A partir de estos resultados identificamos los materiales de interés para la investigación.

En particular, fue necesario elegir estudios que presentaran los instrumentos utilizados para abordar la competencia digital docente en Educación Superior. Así, de los 55 documentos registrados sobre competencia digital del profesorado universitario en el ámbito internacional nos enfocamos en los 30 que se referían a lberoamérica debido al carácter contextual de la CDD y al enfoque biográfico-narrativo de la investigación. Entonces usamos los siguientes criterios de inclusión: actualidad (estudios realizados en los últimos seis años), relevancia (formar parte de proyectos en universidades o ser referencia para otros estudios) y pertinencia (enfocados al perfil docente universitario o usando el método biográfico-narrativo).

Así, se seleccionaron seis estudios realizados en México, España y Chile (Agreda, Hinojo y Sola, 2016; Carrera y Coiduras, 2012; Durán et al., 2016b; Rangel, 2016; Pozos, 2015; Raquimán, 2013). Cinco de estos estudios utilizaron el cuestionario como técnica principal de investigación, y como complemento, pruebas prácticas o bien entrevistas con docentes o con otros participantes de la comunidad universitaria. En el caso de Raquimán (2013), la entrevista en profundidad fue la principal técnica de investigación.

La técnica del análisis de contenido con un enfoque descriptivo y sintético se utilizó tanto para la revisión de los marcos de CDD del profesorado universitario como para los instrumentos. Un complemento importante a nivel conceptual fueron las áreas de CDD propuestas por el INTEF (2013), por su adecuación para clasificar los usos educativos de las tecnologías digitales. Para el análisis de los instrumentos utilizados por los estudios seleccionados se utilizó una matriz con las siguientes categorías: información del estudio, instrumento(s) y base conceptual, dimensiones de la CDD, diseño de instrumento e indicadores, y niveles, escalas y validación. Luego se procedió a la revisión sintética de los indicadores y su reorganización para delimitar la CDD docente y elegir los temas para el guion de entrevista.

A continuación, se presentan tres apartados que sintetizan los hallazgos del análisis, así como el listado de dimensiones, categorías e indicadores para la elaboración del guion de entrevista.

\section{RESULTADOS}

\subsection{Delimitación de la CDD del profesorado Universitario}


En primer lugar, se reconocieron los aspectos clave para delimitar el acercamiento a la CDD del profesorado universitario (tabla 1). Por un lado, confirmamos la pluralidad de aproximaciones a la competencia digital en general y a la CDD del profesorado universitario. Por otro, resaltamos la coincidencia entre los marcos y estudios señalados al identificar conocimientos, habilidades, valores y actitudes que se organizan de determinada manera con el fin de diagnosticar, evaluar o propiciar el desarrollo de dicha competencia. En el caso de los estudios analizados se identifican varias tendencias de agrupación: por áreas (INTEF, 2013, 2017; Durán, et al., 2016a), por unidades competenciales (Pozos, 2015) y por niveles de apropiación competencial (Prendes, 2010; Durán et al., 2016a).

En segundo lugar, se identificaron las líneas específicas de la CDD en relación a la docencia universitaria (tabla 1), además de las áreas de la competencia digital general relacionadas con el desempeño profesional de los docentes, como lo plantean Durán et al. (2016).

Tabla 1. Categorías generales de la CDD del profesorado universitario

\begin{tabular}{|c|c|c|}
\hline & Categorías & Descriptores \\
\hline \multirow{4}{*}{$\begin{array}{l}\text { Ejes de la docencia } \\
\text { universitaria } \\
\text { Fuentes: Durán et } \\
\text { al. (2016a); Pozos } \\
\text { (2015); y análisis de } \\
\text { instrumentos. }\end{array}$} & Pedagógica & $\begin{array}{l}\text { Valoración del potencial didáctico de las TIC, selección y/o } \\
\text { creación de recursos educativos. Diseño de ambientes de } \\
\text { aprendizaje, desarrollo (orientación, tutoría) y evaluación. } \\
\text { Exploración de nuevas metodologías y relación con otros } \\
\text { participantes de la red y del contexto social para el aprendizaje y } \\
\text { la construcción de conocimiento. Ayuda para que los estudiantes } \\
\text { se apropien de las TIC y reflexionen acerca de su uso. }\end{array}$ \\
\hline & $\begin{array}{l}\text { Gestión } \\
\text { educativa }\end{array}$ & Gestión administrativa de las tareas docentes e institucionales. \\
\hline & Investigación & $\begin{array}{l}\text { Uso de herramientas para la investigación y participación en } \\
\text { proyectos de investigación sobre educación y TIC. }\end{array}$ \\
\hline & $\begin{array}{l}\text { Desarrollo } \\
\text { profesional }\end{array}$ & $\begin{array}{l}\text { Reflexión individual o en contextos colectivos, aprendizaje y el } \\
\text { crecimiento profesional, mejora continua del docente, colaboración } \\
\text { con otros además de compartir o difundir buenas prácticas. } \\
\text { Participación en proyectos de investigación o de innovación } \\
\text { educativa con el uso de TIC. }\end{array}$ \\
\hline \multirow{3}{*}{$\begin{array}{l}\text { Áreas de la } \\
\text { competencia } \\
\text { digital general } \\
\text { relacionadas } \\
\text { con la docencia } \\
\text { universitaria }\end{array}$} & Informacional & $\begin{array}{l}\text { Manejo, evaluación y gestión de la información para las } \\
\text { asignaturas o bien para el aprendizaje y desarrollo profesional. }\end{array}$ \\
\hline & $\begin{array}{l}\text { Comunicativo- } \\
\text { colaborativa }\end{array}$ & $\begin{array}{l}\text { Trabajo colaborativo, comunicación e interacción y creación de } \\
\text { redes de colaboración e intercambio. }\end{array}$ \\
\hline & $\begin{array}{l}\text { Creación de } \\
\text { contenidos }\end{array}$ & $\begin{array}{l}\text { Expresión en distintos medios y formatos, estrategias y acciones } \\
\text { para compartir y/o crear contenidos en la red. }\end{array}$ \\
\hline \multirow{2}{*}{$\begin{array}{l}\text { Fuentes: } \\
\text { INTEF (2013); } \\
\text { Durán et al. (2016a); } \\
\text { Pozos (2015); y } \\
\text { análisis de } \\
\text { instrumentos. }\end{array}$} & $\begin{array}{l}\text { Ética, salud y } \\
\text { seguridad }\end{array}$ & $\begin{array}{l}\text { Uso seguro y responsable de las TIC en temas como derechos de } \\
\text { autor, uso de recursos y software abierto, cuidado de la salud, } \\
\text { medio ambiente e impacto del uso de TIC en la sociedad. Actitud } \\
\text { ante el uso, el impacto y el aprendizaje continuo de las TIC. Ayuda } \\
\text { para que los estudiantes reconozcan esta área de la competencia } \\
\text { digital. }\end{array}$ \\
\hline & Técnica & $\begin{array}{l}\text { Conocimientos básicos y técnicos acerca de las TIC. Resolución } \\
\text { de problemas. }\end{array}$ \\
\hline
\end{tabular}

Después, la revisión de literatura sobre la conceptualización de la CDD en general confirmó la importancia de las áreas pedagógico y ética que encontramos presentes en los estudios y modelos analizados. También observamos que el componente actitudinal y reflexivo del docente ante el uso de las TIC se presenta, bien explícito en algunos descriptores o bien de manera implícita como un aspecto transversal.

\subsection{Aspectos para comprender la evolución de la CDD}

Con respecto al DPD, la trayectoria profesional y el perfil docente fueron considerados para contextualizar la evolución de dicha competencia. Por ejemplo, la exploración de los recursos digitales para la docencia puede tener un sentido relacionado con antecedentes que influyen el perfil docente (Sallé y Elgue, 2014). Los instrumentos analizados consideraron puntos relacionados con estos elementos como: los datos sobre la carrera docente, el tipo de adscripción, responsabilidades y experiencia en la docencia y en asignaturas en modalidades 
en línea. El trabajo de Raquimán (2013) incluyó un análisis de los puntos de quiebre en la trayectoria de los docentes y su relación con las TIC para la práctica educativa.

Otra línea relevante para la investigación son los ámbitos que influyen en la CDD. Esto incluye la formación inicial y continua del docente y las brechas que pueden existir entre ambas (Pozos, 2015; Tejada y Pozos, 2018). El impacto de los programas de formación docente es un tema de exploración (Zabalza, 2003; Tejada y Pozos, 2018) que los instrumentos analizados abordan. Ahora bien, la CDD puede verse nutrida por ámbitos no formales e informales, no sólo de la oferta estructurada de formación docente (Tejada y Pozos, 2018), como la familia o el entorno social (Raquimán, 2013).

Otro aspecto más concreto que apareció fue la identificación de recursos digitales relevantes para la docencia, al que sumamos la exploración de los espacios de trabajo de los docentes, en otras palabras, del cuarto propio conectado (Zafra, 2010). Así también, los instrumentos recuperan la autopercepción de los docentes en cuanto a las áreas de competencia digital y sus necesidades de aprendizaje.

Además, los instrumentos incluyeron preguntas semiestructuradas o abiertas sobre los aspectos que los docentes consideran que favorecen el uso de TIC, los obstáculos y propuestas para la mejora. En esta línea merece la pena considerar las problemáticas, dificultades o errores en esa evolución competencial (UNESCO, 2011), las barreras para el aprovechamiento de las tecnologías digitales (Birch y Burnett, 2009; Gutiérrez-Santiuste, Gámiz-Sánchez y Gutiérrez-Pérez, 2015) y las complicaciones que la digitalización puede traer a la labor docente (Selwyn, 2016). Estos puntos subrayan el carácter dinámico y reflexivo del proceso de desarrollo competencial.

\subsection{Dimensiones para el guion de la entrevista}

A partir de la revisión descrita anteriormente se integró un listado de dimensiones, categorías y sus respectivos indicadores para la elaboración del guion de entrevista (tabla 2).

Tabla 2. Dimensiones, categorías e indicadores de la CDD en Educación Superior

\begin{tabular}{|c|c|c|c|}
\hline Dimensión & & Categorías & Indicadores \\
\hline \multirow{3}{*}{$\begin{array}{l}\text { 1. Desarrollo } \\
\text { profesional docente }\end{array}$} & 1.1 & $\begin{array}{l}\text { Trayectoria } \\
\text { profesional }\end{array}$ & $\begin{array}{l}\text { - Razones para elegir la docencia como profesión } \\
\text { - Evolución de la carrera profesional } \\
\text { - Incidentes críticos }\end{array}$ \\
\hline & 1.2 & Perfil profesional & $\begin{array}{l}\text { - Autopercepción y motivaciones profesionales } \\
\text { - Rasgos del conocimiento pedagógico y disciplinar } \\
\text { - Entorno socio-profesional }\end{array}$ \\
\hline & 1.3 & $\begin{array}{l}\text { Perspectiva de } \\
\text { futuro }\end{array}$ & $\begin{array}{l}\text { - Valoración del momento presente } \\
\text { - Metas de desarrollo profesional } \\
\text { - Prospectiva de la universidad, la sociedad y las TIC }\end{array}$ \\
\hline \multirow{5}{*}{$\begin{array}{l}\text { 2. Evolución de la } \\
\text { CDD }\end{array}$} & 2.1 & $\begin{array}{l}\text { Desarrollo de la } \\
\text { CDD) }\end{array}$ & $\begin{array}{l}\text { - Primeras experiencias para la adquisición de la CDD } \\
\text { - Hitos para el desarrollo de la CDD } \\
\text { - Dificultades en relación a la competencia digital } \\
\text { - Influencia de otros ámbitos (familiar, social, laboral) }\end{array}$ \\
\hline & 2.2 & $\begin{array}{l}\text { Uso de TIC en los } \\
\text { ejes de la docencia } \\
\text { universitaria }\end{array}$ & $\begin{array}{l}\text { - Docencia } \\
\text { - Investigación } \\
\text { - Gestión } \\
\end{array}$ \\
\hline & 2.3 & $\begin{array}{l}\text { Aprendizaje } \\
\text { continuo }\end{array}$ & $\begin{array}{l}\text { - Exploración sobre educación y TIC } \\
\text { - Formación continua } \\
\text { - Colaboración (docentes, estudiantes, comunidad) } \\
\text { - Proyectos de investigación o de innovación }\end{array}$ \\
\hline & 2.4 & $\begin{array}{l}\text { Rutinas, espacios } \\
\text { de trabajo y } \\
\text { recursos digitales }\end{array}$ & $\begin{array}{l}\text { - Actividades y uso del tiempo en la jornada laboral } \\
\text { - Espacios de trabajo } \\
\text { - Recursos digitales } \\
\text { - Emociones de las experiencias en red }\end{array}$ \\
\hline & 2.5 & $\begin{array}{l}\text { Uso de TIC en la } \\
\text { vida cotidiana }\end{array}$ & $\begin{array}{l}\text { - Finalidades } \\
\text { - Relación con su competencia digital docente }\end{array}$ \\
\hline
\end{tabular}




\begin{tabular}{|c|c|c|c|}
\hline \multirow{6}{*}{ 3. Áreas de la CDD } & 3.1 & Pedagógica & $\begin{array}{l}\text { - Planificación de experiencias de aprendizaje } \\
\text { - Desarrollo de experiencias de aprendizaje } \\
\text { - Evaluación } \\
\text { - Valoración del potencial del uso de TIC en educación }\end{array}$ \\
\hline & 3.2 & Informacional & $\begin{array}{l}\text { - Fuentes de información y recursos para la docencia } \\
\text { - Búsqueda y selección de información } \\
\text { - Organización y recuperación de información } \\
\text { - Generación de conocimiento }\end{array}$ \\
\hline & 3.3 & $\begin{array}{l}\text { Comunicativo- } \\
\text { colaborativa }\end{array}$ & $\begin{array}{l}\text { - Comunicación en red } \\
\text { - Colaboración en red } \\
\text { - Netiqueta } \\
\text { - } \text { Pesticipación ciudadana } \\
\text { - Gestión la identidad digital }\end{array}$ \\
\hline & 3.4 & $\begin{array}{l}\text { Creación de } \\
\text { contenido digital }\end{array}$ & $\begin{array}{l}\text { - Creación de material digital (propio, integración) } \\
\text { - Uso de licencias y derechos de autor } \\
\text { - Adecuación de recursos digitales } \\
\text { - Programación }\end{array}$ \\
\hline & 3.5 & $\begin{array}{l}\text { Ética, salud y } \\
\text { seguridad }\end{array}$ & $\begin{array}{l}\text { - Valores en las prácticas digitales } \\
\text { - Equidad de acceso y uso de TIC } \\
\text { - Hábitos saludables y sostenibles de uso de TIC } \\
\text { - Protección de datos y dispositivos } \\
\text { - Protección del medio ambiente } \\
\text { - Relación entre sociedad y TIC }\end{array}$ \\
\hline & 3.6 & Técnica & $\begin{array}{l}\text { - Comprensión a nivel usuario de software, hardware y } \\
\text { manejo de dispositivos externos } \\
\text { - Comprensión de las funciones y la estructura de Internet } \\
\text { - Identificación de la infraestructura de los recursos digitales } \\
\text { - Resolución de problemas técnicos }\end{array}$ \\
\hline \multirow{3}{*}{$\begin{array}{l}\text { 4. Valoración crítica } \\
\text { del uso de TIC en } \\
\text { Educación Superior }\end{array}$} & 4.1 & $\begin{array}{l}\text { Cambios y } \\
\text { reflexiones sobre su } \\
\text { docencia }\end{array}$ & $\begin{array}{l}\text { - Cambios en el perfil docente } \\
\text { - Problemáticas del uso de TIC } \\
\text { - Reflexiones para el uso crítico de TIC } \\
\text { - Vías para compartir su experiencia } \\
\text { - Necesidades de aprendizaje en relación a la CDD }\end{array}$ \\
\hline & 4.2 & $\begin{array}{l}\text { La competencia } \\
\text { digital de los } \\
\text { estudiantes }\end{array}$ & $\begin{array}{l}\text { - Importancia en su formación } \\
\text { - Desarrollo de la CDD en la universidad } \\
\text { - Necesidades de aprendizaje }\end{array}$ \\
\hline & 4.3 & $\begin{array}{l}\text { Educación Superior } \\
\text { y cultura digital }\end{array}$ & $\begin{array}{l}\text { - Influencia de la política educativa en su práctica } \\
\text { - Percepción de la competencia digital en su institución } \\
\text { - Impacto de la cultura digital en la Educación Superior y } \\
\text { viceversa } \\
\text { - Desafíos para el uso crítico de TIC en la Educación } \\
\quad \text { Superior } \\
\end{array}$ \\
\hline
\end{tabular}

\section{DISCUSIÓN Y CONCLUSIONES}

Las dimensiones, categorías e indicadores aquí presentados son una vía para comprender la evolución de la CDD en Educación Superior. Por una parte, se reconoce el vínculo de esta competencia profesional con la trayectoria del docente, su perfil y visión del futuro. Por otro, las experiencias significativas para el aprovechamiento de tecnologías digitales en los ejes de la docencia pueden contener claves para la comprensión de dicha evolución. A su vez, la segunda y la tercera dimensión contribuyen a caracterizar esta competencia. Por último, la cuarta dimensión ahonda en el impacto de dicha competencia en el perfil del docente, en su percepción de la competencia digital de los estudiantes universitarios y de la relación entre la Educación Superior y la cultura digital. No obstante, será necesario completar este acercamiento con otras fuentes de evidencia sobre los cambios en la CDD y en el DPD.

Los modelos de CDD del profesorado universitario y los instrumentos del contexto iberoamericano fueron un punto de partida pertinente para identificar categorías relevantes para la investigación. Además, descubrimos una importante coincidencia entre la síntesis aquí presentada sobre la CDD en Educación Superior y la realizada por Durán et al. (2016a). Es importante destacar que los ejes de la docencia que más descriptores agruparon fueron el pedagógico y el desarrollo profesional; la gestión y la investigación fueron tratadas en términos generales. Luego, se descubre la oportunidad de detallar estos ejes, además de la vinculación 
y transferencia del conocimiento. A saber, la programación es una habilidad que se destaca en el modelo del INTEF y que no encontramos que tuviera una presencia importante en los estudios revisados.

La cantidad de indicadores del listado puede representar una limitación debido a que la técnica de entrevista en profundidad requiere la apertura a la expresión de los participantes; no se pretende abarcar todos los indicadores y es probable que el listado presente elementos de carácter analítico. Por lo tanto, se identificó la necesidad de un estudio de obtención de evidencias de validez de contenido de este listado, mediante la evaluación por juicio de expertos (Cabero y Barroso, 2013; Cabero y Llorente, 2013). Esta será una estrategia para cuidar el rigor y la validez en la investigación cualitativa porque implica compartir el sustento teórico y el diseño de la investigación, identificar posibles sesgos e incorporar las observaciones de otros investigadores (Hernández-Sampieri, Fernández y Baptista, 2014)

Otra limitación es el enfoque de CDD elegido pues no se contemplan otras perspectivas como la de múltiples alfabetizaciones (Larraz, 2013) o el modelo TPACK que ha sido adaptado al ámbito universitario (Cejas, Navío y Barroso, 2016). Asimismo, este es un campo en movimiento continuo, con el desarrollo y la publicación de modelos y experiencias.

En general, se confirma la necesidad de profundizar en la comprensión de la CDD desde un enfoque que busca delimitar su abordaje más que encontrar un significado esencial y único, como señalan Carrera y Coiduras (2012), y que reconoce desempeños o aspectos generales de la competencia en lugar de la mera referencia al uso de herramientas digitales. Las distintas propuestas enfatizan la importancia del área pedagógica al reconocer dos responsabilidades centrales para el docente: favorecer el aprendizaje de los estudiantes y propiciar que éstos desarrollen su competencia digital.

Tanto los modelos como los estudios analizados coinciden en la estrategia de fundamentación empleada: la revisión de uno o varios modelos del ámbito nacional e internacional sobre el rol del profesorado en el uso de TIC y de investigaciones en el área, para realizar su propia adaptación. Es decir, los modelos generales son marcos de orientación que requieren su contextualización al perfil docente a quien va dirigido o a las características del proyecto a desarrollar. En este sentido cabe la pregunta por el alcance de la selección de categorías que aquí se presenta para abordar la evolución de la CDD en Educación Superior en otros contextos.

Finalmente, a partir de esta selección de categorías y en línea con los marcos y estudios revisados, es posible concluir que existen aspectos relevantes para la mejora de esta competencia: las actitudes, la reflexión y participación del profesorado, la búsqueda de estrategias para diagnosticar las necesidades formativas relacionadas con la CDD, y el reconocimiento de la influencia del contexto institucional y social que enmarca las prácticas educativas y, por tanto, el desarrollo de esta competencia.

\section{RECONOCIMIENTOS}

La estancia para los estudios de doctorado cuenta con el apoyo del Programa Becas en el Extranjero del Consejo Nacional de Ciencia y Tecnología (CONACYT) de México.

\section{REFERENCIAS BIBLIOGRÁFICAS}

Abrahão, M.H.M.B. y Bolívar, A. (Org.), (2014). La investigación (auto)biográfica en educación. Miradas cruzadas entre Brasil y España. Porto Alegre y Granada: EDIPUCRS y EUG. 
Agreda, M., Hinojo, M. J. y Sola, M. J. (2016). Diseño y validación de un instrumento para evaluar la competencia digital de los docentes en la Educación Superior española. PíxelBit. Revista de Medios y Educación, 49, 39-59. doi: 10.12795/pixelbit.2016.i49.03

Ala-Mutka, K. (2011). Mapping digital competence: towards a conceptual understanding. Sevilla: Joint Research Center. Recuperado de: http://ftp.jrc.es/EURdoc/JRC67075 TN.pdf

Alexander, B., Adams Becker, S., Cummins, M., and Hall Giesinger, C. (2017). Digital Literacy in Higher Education, Part II: An NMC Horizon Project Strategic Brief. Texas: The New Media Consortium.

Amorós, L. (2013). Aproximación a la competencia digital. El rol docente en el trabajo con TIC. Revista d'Innovació Docent Universitària, (5), pp. 98-109.

Birch, D. y Burnett, B. (2009). Bringning academics on board: Encouraging institution-wide diffusion of e-learning environments. Australasian Journal of Educational Technology, 25(1), 117-134.

Bolívar, A., Domingo, J. y Fernández, M. (2001). La investigación biográfico-narrativa en educación. Enfoque y metodología. España: La muralla.

Cabero, J. y Barroso, J. (2013). La utilización del juicio de experto para la evaluación de TIC: el coeficiente de competencia experta. Bordon. Revista de Pedagogía, 65(2), 25-38.

Cabero, J. y Llorente, M. (2013). La aplicación del juicio de experto como técnica de evaluación de las Tecnologías de Información y Comunicación (TIC). Revista de Tecnología de Información y Comunicación en Educación, 7(2), 11-22.

Calvani, A., Cartelli, A., Fini, A. y Ranieri, M. (2008). Models and instruments for assessing digital competence at school. Journal of E-learning and Knowledge Society, 4(3), 183193.

Carrera, F. X. y Coiduras, J. L. (2012). Identificación de la competencia digital del profesor universitario: un estudio exploratorio en el ámbito de las Ciencias Sociales. REDU. Revista de Docencia Universitaria, 10(2), pp. 273-298.

Castañeda, L., Esteve, F. y Adell, J. (2018). ¿Por qué es necesario repensar la competencia docente para el mundo digital? RED. Revista de Educación a Distancia, (56), 1-20. doi: $10.6018 / \mathrm{red} / 56 / 6$

Cejas, R., Navío, A. y Barroso, J. M. (2016). Las competencias del profesorado universitario desde el modelo TPACK (conocimiento tecnológico y pedagógico del contenido). PíxelBit. Revista de Medios y Educación, 49, 105-119. doi: 10.12795/pixelbit.2016.i49.07

Cobo, C. (2016). La Innovación Pendiente. Reflexiones (y provocaciones) sobre educación, tecnología y conocimiento. Montevideo: Colección Fundación Ceibal/Debate. Recuperado de: http://innovacionpendiente.com/

Coll, C. (2007). TIC y prácticas educativas: realidades y expectativas. [Ponencia magistral]. XXII Semana Monográfica de Educación. Madrid: Fundación Santillana. Recuperado de: http://www.oei.es/tic/santillana/coll.pdf

Connelly, M. F., y Clandinin, J. D. (1995). Relatos de la experiencia e investigación narrativa. En Larrosa, J., Arnaus, R., Ferrer, V., Pérez de Lara, N., Connelly, M.F., Clandinin, D. J. y Greene, M. Déjame que te cuente: Ensayos sobre narrativa y educación (pp. 11-59). Barcelona: Laertes.

Durán, M., Gutiérrez, I. y Prendes, M. P. (2016a). Análisis de modelos de competencia digital del profesorado universitario. Revista Latinoamericana de Tecnología Educativa (RELATEC), 15(1), 97-114. doi: 10.17398/1695-288X.15.1.97

Durán, M., Gutiérrez, I. y Prendes, M. P. (2016b). Certificación de la competencia TIC del profesorado universitario: Diseño y validación de un instrumento. Revista Mexicana de Investigación Educativa, 21(69), 527-556.

Fernández, M. (1995). Ciclos en la vida profesional de los profesores. Monográfico. Revista de educación, (306), 153-203. 
Fernández, M. (2006). Desarrollo profesional docente. Granada: Grupo Editorial Universitario.

Fernández, M. (2014). Modelo autobiográfico-narrativo de formación y desarrollo profesional docente. En Abrahão, M.H.M.B. y Bolívar, A. (Org.), La investigación (auto)biográfica en educación. Miradas cruzadas entre Brasil y España (pp. 58-84). Porto Alegre y Granada: EDIPUCRS y EUG.

Flores, G., y Porta, L. (2012). Valores morales en la Educación Superior. Abordaje biográficonarrativo desde profesores universitarios memorables. Revista Digital de Investigación en Docencia Universitaria. 6(1), 40-59.

Gallardo-Echenique, E. (2013). Competencia digital: revisión integradora de literatura. Revista de Ciencias de la Educación ACADEMICUS, 1(3), 56-62.

Gallardo-Echenique, E., Minelli de Oliveira, J., Marqués-Molias, L., y Esteve-Mon, F. (2015). Digital competence in Knowledge Society. Journal of Online Learning and Teaching, 11(1), 1-16.

Gisbert, M., González, J. y Esteve, F. (2016). Competencia digital y competencia digital docente: una panorámica sobre el estado de la cuestión. RIITE. Revista Interuniversitaria de Investigación en Tecnología Educativa, 0, 74-83. doi: 10.6018/riite/2016/257631

Goodson, I. F. (2004). Historias de vida del profesorado. Barcelona: Octaedro.

Gutiérrez-Santiuste., E., Gámiz-Sánchez, V. M., y Gutiérrez-Pérez, J. (2015). Mooc \& Blearning: Students' barriers and satisfaction in formal and non-formal learning environments. Journal of Interactive Online Learning, 13(3), 88-111.

Hernández-Sampieri, R., Fernández, C. y Baptista, M. P. (2014). Metodología de la investigación. Madrid: McGraw-Hill.

Instituto Nacional de Tecnologías Educativas y Formación del Profesorado [INTEF]. (2013). Marco común de competencia digital docente V. 2.0. Plan de cultura digital en la escuela. Recuperado http://educalab.es/documents/10180/12809/MarcoComunCompeDigiDoceV2.pdf

Instituto Nacional de Tecnologías Educativas y Formación del Profesorado [INTEF]. (2017). Marco común de competencia digital docente. Recuperado de: http://blog.educalab.es/intef/2016/12/22/marco-comun-de-competencia-digital-docente2017-intef/

International Society for Technology in Education [ISTE]. (2008). ISTE Standards for Teachers. Recuperado de: http://www.iste.org/standards/iste-standards/standards-forteachers

Janssen, J., Stoyanov, S., Ferrari, A., Punie, Y., Pannekeet, K. y Sloep, P. (2013). Experts' views on digital competence: Commonalities and differences, Computers \& Education, 68, 473-481. doi: 10.1016/j.compedu.2013.06.008

Kelchtermans, G. (1999). Narrative-Biographical Research on Teachers' Professional Development: Exemplifying a Methodological Research Procedure. Annual Meeting of the American Educational Research Association (AERA-symposium).

Larraz, V. (2013). La competència digital a la Universitat. (Tesis para obtener el grado de doctora). Universidad de Andorra: Andorra. Recuperada de: http://www.tdx.cat/handle/10803/113431

Mengual, A. y Roig, R. (2012). La enseñanza y las competencias TIC en el contexto universitario. En Sandoval, Y., Arenas, A., López, E., Cabero, J. y Aguaded, J.I. (Coords.), Las tecnologías de la información en contextos educativos: nuevos escenarios de aprendizaje (pp. 17-32). Colombia: Universidad Santiago de Cali.

Organización de las Naciones Unidas para la Educación, la Ciencia y la Cultura [UNESCO]. (2011). UNESCO ICT competency framework for teachers. París: UNESCO.

Pozos, K. V. (2015). Evaluación de necesidades de formación continua en competencia digital del profesorado universitario mexicano para la sociedad del conocimiento. (Tesis para obtener el grado de Doctora en Calidad y Procesos de Innovación Educativa). 
Universidad Autónoma de Barcelona: España. Recuperado de: http://hdl.handle.net/10803/382466

Prendes, M. P. (Dir.). (2010). Competencias TIC para la docencia en la universidad pública española: Indicadores y propuestas para la definición de buenas prácticas. Informe del proyecto Estudio y Análisis. (MEC), (EA2009-0133).

Prendes, M. P. y Gutiérrez, I. (2013). Competencias tecnológicas del profesorado en las universidades españolas. Revista de Educación, 36(1), 196-222. doi: 10.4438/1988592X-RE-2011-361-140

Rangel, A. (2016). Competencias docentes digitales. Propuesta de un perfil. Píxel-Bit. Revista de Medios y Educación, 46, 235-248. doi: 10.12795/pixelbit.2015.i46.15

Raquimán, P. (2013). Desde sus propias voces: representaciones sobre el cambio en las prácticas pedagógicas de los profesores de ETP en el uso de las TIC: una aproximación a partir de los relatos de vida profesional. (Tesis para obtener el grado de Doctora en Ciencias de la Educación). Universidad de Rouen, Francia, y Pontificia Universidad Católica de Chile: Chile.

Redecker, C. (2017). European Framework for the Digital Competence of Educators: DigCompEdu. Sevilla: Joint Research Centre.

Rodríguez, G., Flores, J. G. y García, E. (1996). Métodos de Investigación Cualitativa. En Metodología de la Investigación Cualitativa (pp. 39-60). Málaga: Aljibe.

Romero-López, M. A. (2017). European Higher Education Area-Driven Educational Innovation. Procedia-Social and Behavioral Sciences, 237, 1505-1512. doi: 10.1016/j.sbspro.2017.02.237

Sallé, M. C. y Elgue, M. (2014). Las TIC en la enseñanza. Un estudio de casos desde el enfoque biográfico-narrativo. EDUCAR, 50, 83-101. doi: 10.5565/rev/educar.114

San Nicolás, M., Vargas, E. y Area, M. (2012). Competencias digitales del profesorado y alumnado en el desarrollo de la docencia virtual. El caso de la Universidad de La Laguna. Revista Historia de la Educación Latinoamericana, 14(19), 227-245. doi: 10.9757/Rhela.19.10

Santos Guerra, M. Á. (2010). La formación del profesorado en las instituciones que aprenden. Revista Interuniversitaria de formación del profesorado, 68(24,2), 175-200.

Selwyn, N. (2014). Internet y educación. En C@mbio. 19 ensayos fundamentales sobre cómo internet está cambiando nuestras vidas (pp. 191-215). España: Open Mind BBVA.

Selwyn, N. (2016). Profesores y tecnología: repensar la digitalización de la labor docente. Boletín de la Institución Libre de Enseñanza, (104), 27-36.

Tejada, J. y Pozos, K. V. (2018). Nuevos escenarios y competencias digitales docentes: Hacia la profesionalización docente con TIC. Profesorado, Revista de Currículum y Formación del Profesorado, 22(1), 41-67.

Veletsianos, G., Kimmons, R. y French, K. (2013). Instructor experiences with a social networking site in a higher education setting: Expectations, frustrations, appropriation, and compartmentalization. Educational Technology Research \& Development, 61(2), 255-278. doi: 10.1007/s11423-012-9284-z

Vuorikari, R., Punie, Y., Carretero Gomez S. y Van den Brande, G. (2016). DigComp 2.0: The Digital Competence Framework for Citizens. Update Phase 1: The Conceptual Reference Model. Luxembourg: European Union. doi:10.2791/11517

Zabalza, M. Á. (2003). Competencias docentes del profesorado universitario: calidad y desarrollo profesional. Madrid: Narcea.

Zafra, R. (2010). Un cuarto propio conectado. (Ciber)espacio y (auto)gestión del yo. Madrid: Fórcola. 


\title{
INFORMACIÓN SOBRE LOS AUTORES
}

\author{
Angelina Lorelí Padilla-Hernández \\ Universidad de Granada
}

Angelina Lorelí Padilla-Hernández es doctoranda en la Universidad de Granada y becaria del Programa Becas en el Extranjero del Consejo Nacional de Ciencia y Tecnología (CONACYT) de México. Licenciada en Ciencias de la Educación por el ITESO, universidad jesuita de Guadalajara, su ciudad natal. Máster en Educación y Gestión del Conocimiento por el ITESO. Tiene experiencia en la formación y asesoría a profesores y comunidad universitaria para el aprovechamiento de plataformas educativas y recursos digitales, y en el diseño de estrategias de gestión del conocimiento. Ha sido tutora de cursos virtuales y docente de grado. Sus líneas de investigación e interés profesional son: la competencia digital, la perspectiva crítica sobre la educación, el uso de tecnologías digitales y la cultura digital, la formación y el desarrollo profesional docente, la gestión del conocimiento, y la formación ciudadana en la educación básica.

Research Gate: https://www.researchgate.net/profile/Angelina Padilla-Hernandez

\section{Vanesa M.. Gámiz-Sánchez}

Universidad de Granada

Vanesa M. a Gámiz Sánchez es Ingeniera en Informática y doctora en Pedagogía por la Universidad de Granada. Desde el año 2010 es profesora del Departamento de Didáctica y Organización Escolar de la Facultad de Ciencias de la Educación en el que anteriormente trabajó como Becaria Predoctoral de Formación de Profesional Investigador. Sus líneas de investigación se orientan hacia el e-learning y la utilización, en general, de las Tecnologías de la Información y la Comunicación en el entorno educativo, especialmente en la Enseñanza Superior. Ha participado como miembro de varios proyectos de innovación docente y coordinado el proyecto titulado "El e-portafolio como estrategia didáctica de aprendizaje y evaluación. Implementación con Mahara integrado con Moodle”. Del mismo modo, ha sido miembro del equipo investigador de varios proyectos europeos sobre e-learning y ha participado como docente en varias actividades de formación en nuevas tecnologías para el profesorado universitario.

Research Gate: https://www.researchgate.net/profile/Vanesa Gamiz-Sanchez

\section{M. a Asunción Romero López Universidad de Granada}

Vicedecana de Practicum de la Facultad de Ciencias de la Educación de la Universidad de Granada y Profesora Titular en el Departamento de Didáctica y Organización Escolar. Licenciada en Pedagogía y Doctora en Ciencias de la Educación por la Universidad de Granada. Miembro del grupo de investigación FORCE (Formación Centrada en la Escuela) de la misma Universidad. Imparte docencia en el área de Didáctica y Organización Escolar. Sus principales publicaciones se centran en la formación docente, tanto inicial como continua, así como el desarrollo profesional del docente y la atención a la diversidad. Algunas de sus publicaciones dentro del ámbito de la mejora educativa son: "Configurando nuevas estrategias para el aprendizaje y la evaluación: el e-portafolio", en J.F. Durán Medina (Coord.). La era de las TT.II.CC. en la nueva docencia, McGraw Hill -2015- Ma Asunción Romero, Vanesa Gámiz y Emilio Crisol; "The European Higher Education Area-Driven Educational Innovation", simposio coordinado en el EDUHEN16 por M"a Asunción Romero López; y "Emotions of university students in the use of digital portfolios as a tool of learning and assessment" -ponencia realizada en el EDULEARN16 Conference- por Vanesa María Gámiz-Sánchez, Emilio Crisol-Moya y Maa Asunción Romero López.

Research Gate: https://www.researchgate.net/profile/Maria Asuncion Lopez

Web personal: http://hum386.ugr.es/\%7Emromero/datos personales/

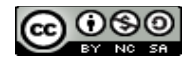

Los textos publicados en esta revista están sujetos a una licencia de Reconocimiento 4.0 España de Creative Commons. Puede copiarlos, distribuirlos, comunicarlos públicamente y hacer obras derivadas siempre que reconozca los créditos de las obras (autoría, nombre de la revista, institución editora) de la manera especificada por los autores o por la revista. La licencia completa se puede consultar en:Licencia Creative Commons Atribución-NoComercialCompartir por igual 4.0 Internacional. 